Published in final edited form as:

Anal Chem. 2020 April 21; 92(8): 6150-6157. doi:10.1021/acs.analchem.0c00831.

\title{
Electrode-Free Concentration and Recovery of DNA at Physiologically Relevant Ionic Concentrations
}

\author{
Sixuan Li, \\ Department of Mechanical Engineering, Johns Hopkins University, Baltimore, Maryland 21218, \\ United States;
}

Andrew Li,

Department of Biomedical Engineering, Johns Hopkins University, Baltimore, Maryland 21218, United States

\section{Kuangwen Hsieh,}

Department of Mechanical Engineering, Johns Hopkins University, Baltimore, Maryland 21218, United States

Sarah M. Friedrich,

Department of Biomedical Engineering, Johns Hopkins University, Baltimore, Maryland 21218, United States;

\section{Tza-Huei Wang}

Department of Mechanical Engineering and Department of Biomedical Engineering, Johns

Hopkins University, Baltimore, Maryland 21218, United States;

\section{Abstract}

Advances in microanalytical and microfluidic technologies have enabled rapid and amplificationfree detection of DNA with a high signal-to-noise ratio. The low sample volume, however, poses a limit in the DNA detection sensitivity, which can be challenging for analyzing rare DNA in physiological samples. One way to improve the sensitivity is to concentrate the DNA in the sample prior to the analysis. The most common DNA concentration techniques are based on electrokinetics, which require an external electric field and generally become ineffective in high ionic concentration conditions. In this work, we present a facile method termed high-salt molecular rheotaxis (HiSMRT) to concentrate and recover DNA from samples with physiologically relevant ionic concentrations without any external electric field. HiSMRT requires only pressure-driven flow and ion concentration gradient to induce a stable local electric field and achieve DNA concentration, making it impervious to high ionic concentrations. We demonstrate

Corresponding Author: Tza-Huei Wang - Department of Mechanical Engineering and Department of Biomedical Engineering, Johns Hopkins University, Baltimore, Maryland 21218, United States; thwang @jhu.edu.

Supporting Information

The Supporting Information is available free of charge at https://pubs.acs.org/doi/10.1021/acs.analchem.0c00831.

Figures of illustrations of setup and procedure, single-molecule chromatograms, single-molecules counts, percentages of fragments, two-dimensional axial-symmetric COMSOL simulations, time traces, fitted linear curves, fluorescence microscopy images, and COMSOL simulation results, table of concentrations, and discussions of COMSOL model of electric field and potential generated by HiSMRT (PDF)

Notes

The authors declare no competing financial interest. 
that HiSMRT performs robustly at ionic concentrations equivalent to $2 \%-20 \%$ of the ionic concentration in blood serum. HiSMRT can concentrate DNA by up to 960 -fold and recover an average of $96.4 \%$ of the DNA fragments from 2.0 to $23 \mathrm{kbp}$ uniformly. The concentration process using HiSMRT takes as little as $7.5 \mathrm{~min}$. Moreover, we show that this technique can be easily integrated to perform DNA concentration, size separation, and single-molecule detection all in one platform. We anticipate that this technique will be applicable to a wide range of biological samples and will help to improve the sensitivity of nucleic acid detection for low-abundance DNA biomarkers.

\section{Graphical Abstract}

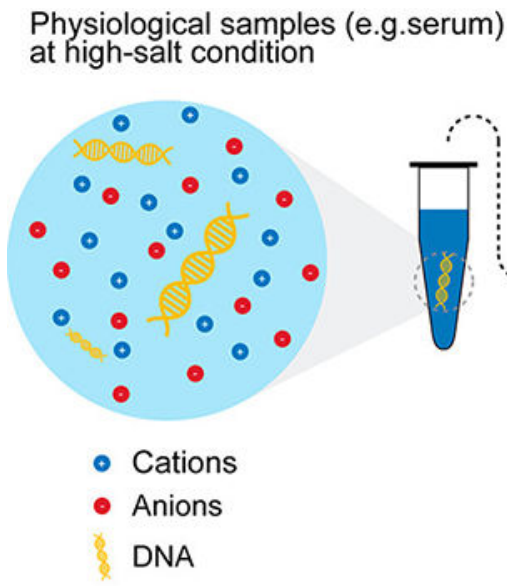

High-Salt Molecular Rheotaxis

Analysis of DNA remains one of the mainstays in biology, medicine, and forensics. ${ }^{1-3}$ To enable highly sensitive DNA assay, conventional analysis generally requires PCR amplification, which has major drawbacks such as prior information on the target sequence, amplification errors, and biases. ${ }^{4}$ More recently, the advent of microanalytical and microfluidic methods ${ }^{5}$ has enabled rapid and accurate DNA detection and quantification while removing the need for amplification, reducing sample and reagent consumption, and improving the signal-to-noise ratio. ${ }^{6}$ Despite these advantages, microfluidic systems can only process small analytical volumes, which is challenging when DNA molecules are present at low concentrations, as is typically the case for clinical samples. ${ }^{7-9}$

Concentrating DNA molecules prior to downstream detection presents a promising approach to increase assay sensitivity. ${ }^{10,11}$ Among the concentration techniques commonly used in microfluidic devices, ${ }^{12}$ electrophoretic methods such as field-amplified sample injection (FASI) and isotachophoresis (ITP) remain the most popular. ${ }^{13-16}$ Most of the electrokinetic methods, including FASI, have limited compatibility with high ionic concentration conditions, as their performance sharply declines with increasing ionic strength. Diluting the sample to lower the ionic concentration is unfavorable in this case, as it will also lower the concentration of the analyte in the sample, thus decreasing detection sensitivity. ${ }^{17}$ As many biological samples, such as blood serum and urine, possess high ionic concentration-based conductivity, ${ }^{18,19}$ they cannot be analyzed by most of the electrophoretic methods. This condition highlights the need for a method to directly concentrate samples with high ionic 
concentrations with minimal dilution in order to detect the ultralow concentration DNA biomarkers (such as circulating tumor DNA). Although ITP has demonstrated concentration enhancement of DNA from biological samples with high-salt concentration, ${ }^{20}$ its on-chip implementation faces practical challenges, including the use of an external high-voltage supply, surface treatment to suppress electro-osmotic flow (EOF), and controlling sample dispersion and joule heating. ${ }^{21-23}$ Furthermore, it is difficult to optimize the performance by simultaneously tuning the parameters, including electric field strength and device geometry, 21 and avoiding DNA aggregation and cleavage in a high-electric field. ${ }^{24}$ Thus, there is a critical need for alternate, electrode-less DNA concentration techniques that can perform in high ionic concentration conditions.

We previously reported a new technique, which we coined molecular rheotaxis (MRT), that can concentrate DNA without applying any external electric field. ${ }^{25}$ MRT leverages a stable salt concentration gradient, established by pressure-driven flow and the difference of the ion diffusivity to generate spontaneous electric field to concentrate DNA. Under the optimized condition, MRT concentrated DNA in a reservoir of deionized (DI) water by nearly 10000 fold. However, MRT has limited capacity for concentration DNA in high ionic concentrations or at least requires significant dilution of samples in physiological salt conditions. Moreover, because MRT relies on a localized electric field, it is inherently challenging to recover the majority of the DNA molecules in the sample reservoir, thus posing additional disadvantages for working with samples with low concentrations of DNA.

In response, we have developed high-salt MRT (HiSMRT), a technique that retains the electrode-free feature of MRT but achieves both concentration and recovery of DNA from samples with physiologically relevant ionic concentrations. HiSMRT readily integrates with single-DNA-molecule analysis platforms (e.g., single-molecule-free solution hydrodynamic separation (SML-FSHS) ${ }^{26,27}$ and cylindrical illumination confocal spectroscopy (CICS) ${ }^{28}$ ), which allows us to characterize the concentration factor and recovery efficiency of HiSMRT with single-molecule sensitivity. We demonstrated that HiSMRT can perform effectively at high ionic concentrations. In buffers ranging from $2 \%$ to $20 \%$ ionic concentration of blood serum equivalence, the performance of HiSMRT remained stable, demonstrating up to 960fold concentration factors and an average of $96.4 \%$ recovery efficiency. Moreover, we investigated the stability of HiSMRT, and in the stable regime, the concentration process takes as short as $7.5 \mathrm{~min}$. To our knowledge, this is the first demonstration of an electrodefree approach for DNA concentration and recovery directly at physiologically relevant ionic concentrations.

\section{EXPERIMENTAL SECTION}

\section{Buffer and Sample Preparation.}

EB-HCl buffer consisted of Bis-Tris, EACA ( $\varepsilon$-aminocaproic acid), and $\mathrm{HCl}$ (hydrochloric acid $)$ at the constant ratio $(1 \times \mathrm{EB}-\mathrm{HCl}=2 \mathrm{mM} \mathrm{EACA}+8 \mathrm{mM}$ Bis-Tris $+1.8 \mathrm{mM} \mathrm{HCl}, \mathrm{pH}$ 7). EACA and Bis-Tris (Sigma-Aldrich) were dissolved in deionized water at $500 \mathrm{mM}$ as the stock concentration to prepare the EB-HCl buffer with stock hydrochloric acid at $6 \mathrm{M}$ (Sigma-Aldrich). EB-HCl buffers with concentration ranging from $1 \times, 2.5 \times, 5 \times$, to $10 \times$ were used as the high-salt buffer. The low-salt buffer was ultrapure water (simplicity water 
purification system, Millipore). Hind III digested $\lambda$ DNA (New England Biolabs) was used as the DNA sample for all experiments. In order to eliminate an additional fragment (27 $\mathrm{kbp}$ ) formed by the annealing of the cohesive ends of fragments 23 and $4.4 \mathrm{kbp}$, the DNA sample was heated at $60{ }^{\circ} \mathrm{C}$ for $3 \mathrm{~min}$, followed by cooling on ice for $3 \mathrm{~min}$. Then, the DNA at $5 \mathrm{ng} / \mu \mathrm{L}$ was stained with $1 \mu \mathrm{M}$ TOTO-3 dye (ThermoFisher Scientific) in the EB-HCl buffer. The DNA sample was stored on ice in a dark environment for at least $1 \mathrm{~h}$ and was further diluted to a final concentration of $5 \mathrm{ng} / \mathrm{mL}$ in the EB-HCl buffer before each run. Alexa 647-NHS ester (ThermoFisher Scientific) stocked at $1 \mu \mathrm{M}$ was spiked in the blank EB-HCl buffer at $1 \mathrm{nM}$ and used as a "free dye" marker for flow velocity calibration.

\section{Microcapillary Preparation.}

A fused silica microcapillary (Polymicro Technologies, Molex) with a inner diameter of 5 $\mu \mathrm{m}$ and a total length of $130 \mathrm{~cm}$ was used. The polyimide coating around the glass capillary was burned and cleaned to get the capillary a transparent observation window at $100 \mathrm{~cm}$ from the concentration chamber end. The new capillary was rinsed with deionized water and EB-HCl buffer for at least $12 \mathrm{~min}$ (>3 column volumes). Next, Alexa 647 dye at $1 \mathrm{nM}$ in EB-HCl buffer $(\sim 28 \mathrm{pL})$ was injected from the concentration chamber, and the retention time it took to pass the detector was used to calibrate the capillary diameter based on the Hagen-oiseuille equation.

\section{Experimental Setup and Procedure.}

The experimental setup has been described in our previous work, ${ }^{25}$ while the experimental procedure was modified as described below. Briefly, both ends of the capillary were housed in pressure chambers. The DNA concentration chamber contained deionized water, while the sample chamber contained the initial low-concentration DNA sample in high-salt EB-HCl buffer. The detection window of the capillary was fixed with epoxy glue onto a glass slide, which sat on top of the cylindrical illumination confocal spectroscopy (CICS) singlemolecule detector. In this study, the operation mode was modified, and the procedures were as follows (Figure S1). First, we filled the entire capillary $(\sim 27 \mathrm{~nL})$ at $800 \mathrm{psi}(3.9 \mathrm{~mm} / \mathrm{s})$ with the TOTO-3 labeled DNA sample at $0.005 \mathrm{ng} / \mu \mathrm{L}$ in EB-HCl buffer. Next, we replaced the DNA sample with a blank EB-HCl buffer and flushed all the DNA in the capillary into the concentration chamber, where the DNA were concentrated. In the last step, a plug of concentrated DNA ( $28 \mathrm{pL})$ was back-injected at $50 \mathrm{psi}$ and single-molecule-free solution hydrodynamic separation (SML-FSHS) was performed at $350 \mathrm{psi}(1.7 \mathrm{~mm} / \mathrm{s})$ for highresolution DNA separation and single-molecule detection in the downstream. To quantify the concentration performance for each DNA fragment in the sample, we performed a standard control experiment with only SML-FSHS by injecting a high-concentration DNA plug at 2.5 $\mathrm{ng} / \mu \mathrm{L}$ with same injection volume $(28 \mathrm{pL})$ at $50 \mathrm{psi}$ and running the SML-FSHS at $350 \mathrm{psi}$. The detailed explanation of the quantification is described in the paragraphs below. All the fluidic operations including sample loading, DNA concentration, back injection, and size separation were performed by applying positive pressure from the sources (purified helium gas, Airgas), controlled by a dual valve pressure regulator (Alicat Scientific). The pressures were set and monitored through a custom LabVIEW program, which allowed for precise sample manipulation and minimal manual input. Additional details of the experimental setup and the SML-FSHS platform are available in the Supporting Information. 


\section{Data Collection and Analysis.}

The single-molecule fluorescence bursts were collected by an avalanche photodiode (APD) detector of the SML-FSHS in $0.1 \mathrm{~ms}$ bin and saved by a custom LabVIEW data acquisition program. A baseline (average background) and a threshold (background plus four standard deviations) were applied to the raw data to count the number of DNA molecules in a custom MATLAB script (R2018b, The Mathworks). The results were plotted as single-molecule chromatogram in $1 \mathrm{~s}$ bin. OriginPro (OriginLab, version 9) was used to fit the peaks in the chromatograms into a set of baseline-subtracted Gaussian peaks. The peak area (area under the curve) was used as the number of molecules for each DNA fragment.

To evaluate the performance of our method, we used two criteria: concentration factor and recovery efficiency. The concentration factor evaluates how many folds the DNA fragments are concentrated by, and it is quantified by comparing it to a standard control at high concentration. For each DNA fragment, the concentration factor (CF) is calculated as

$$
\mathrm{CF}=\frac{N}{N_{\mathrm{c}}} \times D_{\mathrm{F}}
$$

where $N$ is the number of molecules after concentration; $N_{\mathrm{c}}$ is the number of molecules of the standard control; $D_{\mathrm{F}}$ is the dilution factor. In all the experiments, an initial DNA concentration of $5 \mathrm{ng} / \mathrm{mL}$ and a standard control of $2.5 \mathrm{ng} / \mu \mathrm{L}$ were used (Figure S2a), which gave a constant $D_{\mathrm{F}}$ of 500 .

The recovery efficiency evaluates the fraction of DNA molecules that get concentrated, recovered, and delivered back to the capillary versus the input DNA into the capillary. For each fragment, the recovery efficiency (RE) is calculated as

$$
\mathrm{RE}=\frac{N}{N_{\text {input }}} \times 100 \%
$$

where $N_{\text {input }}$ is the number of molecules input in the concentration experiment, and $N_{\text {input }}$ is quantified as

$$
N_{\text {input }}=N_{\text {tot }} \times \frac{N_{\mathrm{c}}}{N_{\mathrm{c}, \text { tot }}}
$$

where $N_{\text {tot }}$ is the total number of molecules including all sizes of fragments input; $N_{\mathrm{c}}$ is the number of molecules of individual size in the standard control (Figure S2b); $N$ c,tot is the total number of molecules including all sizes of fragments in the standard control; $\frac{N_{\mathrm{c}}}{N_{\mathrm{c}, \text { tot }}}$ is the percentage of each fragment in the Hind III digested $\lambda$ DNA sample (Figure S2c).

\section{COMSOL Simulation.}

Two-dimensional axial-symmetric simulations of HiSMRT generated by the salt concentration gradient and ion diffusion difference were performed using a finite-element simulation package (COMSOL Multiphysics 5.2, COMSOL). The model coupled singlephase laminar flow with the Nernst-Planck equations for chemical species transport. The 
electric potential and the electric field strength at various flow velocities and $\mathrm{EB}-\mathrm{HCl}$ buffer concentrations were simulated and evaluated. More detailed descriptions of the model and the parameters are provided in the Supporting Information.

\section{- RESULTS AND DISCUSSION}

\section{Working Principle of HiSMRT.}

HiSMRT concentrates DNA via an electric field locally induced from a stable ion concentration gradient that is created by simply pressurizing a high ionic concentration buffer containing the DNA sample through a capillary into a reservoir of low ionic concentration buffer (Figure 1). This phenomenon occurs at the interface between the two buffers due to (1) the steady pressure-based injection of the high ionic concentration buffer, which establishes a stable ion concentration gradient $(\nabla C)$, and (2) the diffusivity difference between the cation $\left(D_{\text {cation }}\right)$ and the anion $\left(D_{\text {anion }}\right)$ in the high ionic buffer, which generates a spontaneous local electric field $(E)$ as these ions diffuse into the reservoir at different rates. This electric field can reach $>60 \mathrm{~V} / \mathrm{cm}$ (Supporting Informatio Figure S3, COMSOL simulation), which is comparable to the electric fields applied in electrokinetic methods. ${ }^{23,29}$ In this spontaneously induced electric field, the negatively charged DNA molecules experience both electrophoretic force $\left(F_{\mathrm{E}}\right)$ and fluid drag force $\left(F_{\text {Drag }}\right)$, which counterbalance each other and consequently accumulate and become concentrated at the sample outlet of the microcapillary.

Our implementation of HiSMRT, which leverages pressure-driven flow and ion diffusivity difference to generate the ion concentration gradient at the interface between the microcapillary outlet and the large reservoir offers several advantages over previous electrode-free concentration techniques such as diffusiophoresis ${ }^{30}$ and MRT. ${ }^{25}$ In conventional diffusiophoresis-based techniques, the ion concentration gradient is typically generated in a channel ${ }^{31-34}$ or at the interface of an ion-exchange media. ${ }^{35,36}$ These techniques not only induce diffusiophoresis to migrate particles but also cause potentially unwanted diffusioosmosis that convectively drive the fluid flow, ${ }^{34,37,38}$ which can complicate concentration performance. In contrast, by generating the ion concentration gradient at the outlet of the microcapillary, HiSMRT effectively avoids such diffuisoosmotic flow and creates a stable and strong electric field to enable robust DNA concentration. Importantly, whereas it was challenging for MRT to recover the majority of the DNA molecules originally in the reservoir, in HiSMRT, the majority of the DNA molecules could be concentrated and easily recovered through simple back-injection (Figure S1). Finally, the concentrated and recovered DNA can be quantified by fluorescence microscopy, UV absorption meter, or single-molecule detection such as cylindrical illumination confocal spectroscopy (CICS) ${ }^{28}$ HiSMRTcan also be incorporated with analytical chemistry platforms such as capillary electrophoresis (CE) or high-pressure liquid chromatography (HPLC).

\section{Visual Validation of HiSMRT via Fluorescence Microscopy.}

As an initial demonstration, we set up HiSMRT to concentrate fluorescently stained DNA and employ fluorescence microscopy for observation. To do so, we injected Hind III 
digested $\lambda$ DNA in $1 \times \mathrm{EB}-\mathrm{HCl}$ buffer $(\mathrm{pH} 7)$ through a microcapillary into a reservoir of deionized (DI) water. Empirically, $1 \times$ EB-HCl buffer supports robust HiSMRT because the major cation in this buffer, Bis-Tris ${ }^{+}$, diffuses much slower than the major anion, $\mathrm{Cl}^{-}, 39,40$ in the DI water reservoir, which produces a steep ion concentration gradient that induces a strong local electric field. ${ }^{25}$ In this experiment, TOTO-3 DNA intercalating dye was spiked in both $1 \times \mathrm{EB}-\mathrm{HCl}$ buffer and DI water to yield strong fluorescence that can be visualized via fluorescence microscopy. Under these conditions, we observed a fluorescent bolus forming at the outlet of the microcapillary in $300 \mathrm{~s}$ and growing further in size by $600 \mathrm{~s}$ (Figure 2a). These results clearly indicate that fluorescently stained DNA continuously accumulate at the outlet, thus validating that DNA is concentrated by HiSMRT. We subsequently illustrate the essential role of ion concentration gradient in HiSMRT. Here, we repeated the initial HiSMRT experiment but replaced the DI water in the reservoir with $1 \times$ EB-HCl buffer, which eliminates the ion concentration gradient and disables HiSMRT. In the absence of the ion concentration gradient, a fluorescent bolus fails to appear at the outlet of the microcapillary even after $600 \mathrm{~s}$ (Figure 2b), suggesting that DNA can only disperse into the reservoir. Failed concentration of DNA under disabled HiSMRT confirms that the ion concentration gradient created by buffer mismatch (between $1 \times \mathrm{EB}-\mathrm{HCl}$ buffer and DI water) is critical for establishing HiSMRT and concentrating DNA.

\section{Integration of HiSMRT with Hydrodynamic Separation and Single-Molecule Detection.}

Among the various DNA analysis modalities that can be combined with HiSMRT, in this work, we have integrated HiSMRT with single-molecule-free solution hydrodynamic separation (SML-FSHS) ${ }^{26}$-a high-resolution, wide-dynamic-range, size-based DNA separation method driven by pressure $26,27,41$-and cylindrical illumination confocal spectroscopy (CICS) ${ }^{28}$-a high-sensitivity single-molecule detection method. ${ }^{42-44}$ Integration of these modalities as "HiSMRT-(SML-FSHS)-CICS" (hereafter referred to as HSC) allows us to recover the concentrated DNA by HiSMRT and quantify DNA fragments with singlemolecule sensitivity while still operating without applying an external electric field. Specifically, in our setup, we first injected a fluorescently stained DNA sample through a microcapillary into the reservoir to trigger HiSMRT (Supporting Information Figure S1a). After the DNA sample was flushed into the reservoir and concentrated by HiSMRT (Supporting Information Figure S1b), we simply reversed the pressure to "back-inject" and recovered the concentrated DNA into the microcapillary. The DNA plug containing various fragment sizes will be separated into bands via hydrodynamic separation, ${ }^{27}$ where larger DNA fragments migrate faster and are detected at earlier time points by CICS. This results in a trace of DNA fluorescence bursts as a function of retention time, which is recorded as a free solution hydrodynamic separation chromatogram (Supporting Information Figure S1c).

Using our HiSMRT-(SML-FSHS)-CICS setup to concentrate, separate, and analyze DNA, we indeed acquired a time trace (Figure 3a) with distinct clusters of fluorescence bursts that correspond to the known DNA fragment sizes Here, we selected Hind III digested $\lambda$ DNA as we want to demonstrate that HiSMRT can indiscriminately concentrate a wide range of DNA sizes (i.e., 23, 9.4, 6.6, 4.4, 2.3, and $2.0 \mathrm{kbp}$ for Hind III digested $\lambda$ DNA). We subsequently enumerate and sum the fluorescent bursts over periods of $1 \mathrm{~s}$ to plot a singlemolecule chromatogram, which allows us to clearly visualize distinct peaks that represent 
the number of DNA molecules from different fragment sizes (Figure 3b, blue curve). For comparison, we repeated the same experiment without ionic concentration gradient, thus no HiSMRT, and detected only sparse DNA fluorescent bursts in the resulting time trace (Supporting Information Figure S4), which translates to a flat and noisy single-molecule chromatogram (Figure 3b, red curve). The single-molecule chromatogram results, which are drastically different from DNA samples with and without HiSMRT concentration, not only mirror our observation of HiSMRT via fluorescence microscopy but also provide the means for quantitatively analyzing the concentration and recovery performances of HiSMRT.

\section{Performance of HiSMRT at High lonic Concentrations.}

In this work, we measured the concentration factor and the recovery efficiency of HiSMRT from the single-molecule chromatograms to evaluate its performance. The concentration factor is the amount by which the DNA sample is concentrated, and the recovery efficiency is the fraction of input DNA molecules that is recaptured upon concentration (see the "Experimental Section"). We first measured the concentration factor and the recovery efficiency of HiSMRT in $1 \times \mathrm{EB}-\mathrm{HCl}$ buffer. Under this condition, all fragment sizes of Hind III digested $\lambda$ DNA were concentrated by up to 871 folds (Figure $4 a, 1 \times$ ) and were recovered by average of $95.6 \%$ (Figure $4 \mathrm{~b}, 1 \times$ ). HiSMRT also exhibits small DNA sizedependent bias within the size range from 2.0 to $23 \mathrm{kbp}$, as the coefficient of variation (CV) across the fragments are less than $8.4 \%$ and $5.7 \%$ for concentration factor and recovery efficiency, respectively.

Unlike most conventional electrophoretic methods and our previous MRT method that only work effectively with low ionic-conductivity buffers, ${ }^{14-16}$ HiSMRT performs robustly in high ionic concentrations and is consistent across a range of ionic concentrations. As a demonstration, we performed HiSMRT in $2 \times, 5 \times$, and $10 \times$ EB-HCl buffer and measured the concentration factors and the recovery efficiencies under these conditions. We note that the conductivity of $10 \times \mathrm{EB}-\mathrm{HCl}$ buffer, at $0.3 \mathrm{~S} / \mathrm{m}$ (Supporting Information Figure S5), is equivalent to the conductivity of $20 \%$ blood serum or $0.2 \times$ PBS (phosphate-buffered saline). 18,19 To ensure accurate quantification of DNA molecules at increasing ionic concentrations, we accordingly increased the concentration of TOTO-3 dye in the DI water reservoir such that effective TOTO-3 dye staining can be retained (Supporting Information Figure S6 and Table S1). In these buffers with elevated ionic concentrations, HiSMRT remained effective, concentrating Hind III digested $\lambda$ DNA by at least 827 folds (Figure $4 a, 2 \times, 5 \times$, and $10 \times$ ) and recovering at least $94.0 \%$ of input Hind III digested $\lambda$ DNA (Figure $4 b, 2 \times, 5 \times$, and $10 \times$ ). In the best case, a 960-fold concentration factor and $97.6 \%$ recovery efficiency were achieved (Figure 4a, 5×, 2.0 and $2.3 \mathrm{kbp}$ ). These results clearly demonstrate that HiSMRT is robust in at least $10 \times \mathrm{EB}-\mathrm{HCl}$ buffer and consistent across a 10-fold ionic concentration range. Of note, by virtue of the near 1000-fold concentration factor achieved by HiSMRT, the effective sample screening volume of our single-molecule detection system essentially increased by almost 3 orders of magnitude (from $28 \mathrm{pL}$ to $27 \mathrm{~nL}$ ), highlighting the usefulness of HiSMRT.

We further note that HiSMRT can function in even higher ionic concentrations. In principle, higher ionic concentrations should induce steeper ion concentration gradients to concentrate 
DNA. However, electrostatic screening in higher ionic concentrations can cause higher off rates of the TOTO-3 from DNA TOTO-3 dye from staining DNA molecules, leading to a large variance on the number of dye molecules bound to DNA ${ }^{45}$ and thus inaccurate quantification of DNA molecules via CICS (Supporting Information Figure S7). Dyes with slower off rates, such as YOYO-1, may lead to stronger fluorescence signal and more accurate single-molecule quantification. ${ }^{45}$ In a preliminary experiment, we observed via fluorescence microscopy that, even in $30 \times \mathrm{EB}-\mathrm{HCl}$ buffer (i.e., ionic concentration equivalent to $60 \%$ blood serum), Hind III digested $\lambda$ DNA stained with YOYO- 1 could be concentrated by HiSMRT to form a bright bolus at the outlet of the microcapillary (Supporting Information Figure S8). This result shows that HiSMRT can concentrate and recover DNA in a physiological sample with less than 2-fold dilution into the working buffer, which supports that HiSMRT works at the physiologically relevant ionic concentrations. Quantitative characterization by our integrated HSC setup in the future would allow us to evaluate the capacity of HiSMRT in concentrating low-abundance nucleic acid biomarkers from biological samples in physiological conditions.

\section{Performance and Operational Speed of HiSMRT under Various Flow Velocities.}

We further explore the flow velocities that would ensure the performance of HiSMRT. Here, we tested HiSMRT in 10× EB-HCl buffer under five injection pressures resulting in five different flow velocities ranging from 0.53 to $3.74 \mathrm{~mm} / \mathrm{s}$. At the three low flow velocities $(0.53,1.62$, and $2.74 \mathrm{~mm} / \mathrm{s})$, which are denoted as the "stable regime", HiSMRT maintained effective and consistent performance (Figure 5 , green region). At the lowest velocity ( 0.53 $\mathrm{mm} / \mathrm{s}$ ), the highest concentration factor ( $>830$-fold) and recovery efficiency $(>94 \%)$ was observed and the performance was uniform across fragments from 2.0 to $23 \mathrm{kbp}$ with less than $3 \%$ size bias. Increasing the flow velocity to 1.6 and $2.7 \mathrm{~mm} / \mathrm{s}$, the performance decreased marginally by less than $8 \%$ and $7 \%$, respectively. On the contrary, at even higher velocities ( 3.24 and $3.77 \mathrm{~mm} / \mathrm{s}$ ), which are denoted as the "unstable regime", both concentration factors and recovery efficiencies dropped either steadily for large DNA fragments or even more sharply for smaller fragments (Figure 5, red region). At a velocity of $3.77 \mathrm{~mm} / \mathrm{s}$, the concentration factor and the recovery efficiency decreased to as low as 81fold and $9.5 \%$, respectively, which is more than 10 times less than the performance in the "stable regime". This decreasing stability of HiSMRT as a result of increasing flow velocities was visually verified via fluorescence microscopy (Supporting Information Figure S9). In addition to the experimentation, our simulations also reveal that the electric field strength decreases exponentially with the flow velocity (Supporting Information Figure S10a). As a result, lower electrophoretic force and a higher flow drag force disrupt the fore balance on the DNA molecule and HiSMRT becomes unstable. Moreover, we also observed a notable variation of the performance across DNA sizes in addition to the overall lower performance in the "unstable regime". At a velocity of $3.24 \mathrm{~mm} / \mathrm{s}$, the coefficient of variation (CV) across the fragments ranging from 2.0 to $23 \mathrm{kbp}$ are over $45 \%$ and $46 \%$ for concentration factor and recovery efficiency, respectively, while the CV was less than $4.6 \%$ in the stable regime. It is interesting that large DNA renders better performance than smaller DNA. One possible explanation for this size selectivity at high flow velocities is that the force imbalance occurs earlier for smaller DNA: The magnitude of the electrophoretic force on the DNA molecule increases linearly with DNA length. On the contrary, the increase of 
the hydrodynamic radius of DNA molecule with DNA size will slow down and finally plateau at $\sim 100 \mathrm{kbp} .{ }^{46}$ This means that the flow drag force increases with the size slower than that of the electrophoretic force. As a result, smaller DNA will become unstable earlier than the larger DNA. Other factors may contribute to this size variation, including conformation (shape) variation across different length of DNA molecules and the difference in the effective charges at higher ionic concentrations. ${ }^{47}$

In addition to its effect on the concentration and recovery performance of HiSMRT, flow velocity also dictates the operation speed of HiSMRT. To achieve the same throughput of input DNA sample, the concentration time of HiSMRT is inversely proportional to the flow velocity (Supporting Information Figure S10b). For example, at $0.53 \mathrm{~mm} / \mathrm{s}$, it takes $41 \mathrm{~min}$ to concentrate $27 \mathrm{~nL}$ of DNA by up to 855 -fold. In contrast, at $2.74 \mathrm{~mm} / \mathrm{s}$, it takes only 7.5 min to achieve up to 752-fold concentration. These results highlight that the operational parameters of HiSMRT can be flexibly tuned to either maximize concentration factor and recovery rate or minimize the concentration time.

\section{CONCLUSION}

We introduce herein a new technique, which we coined high-salt molecular rheotaxis (HiSMRT), that enables DNA concentration and recovery from physiologically relevant ionic concentration buffer in an electrode-free manner. HiSMRT utilizes pressure-driven flow and buffer mismatch to establish a stable concentration gradient in which the difference of the ion diffusivity induces an electric field to concentrate DNA. The operation only used pressure-driven flow, rendering the combined DNA concentration and size separation experiment truly electrode-free. We have demonstrated the DNA concentration and recovery performance of HiSMRT through two systems. First, we directly observed the concentration process and analyzed the effect of ionic gradient on the DNA concentration via fluorescence microscopy. Second, we integrated HiSMRT to single-molecule-free solution hydrodynamic separation (SML-FSHS) and cylindrical illumination confocal spectroscopy (CICS) to quantitatively analyze its performance. We have demonstrated that HiSMRT achieves highconcentration factors (up to 960-fold), high recovery efficiencies (on average 96.4\%) and minimal DNA size bias and takes as short as $7.5 \mathrm{~min}$. HiSMRT retained effective DNA concentration and recovery in high ionic concentration of up to equivalence of $20 \%$ blood serum and across a 10 -fold change in ionic concentrations.

We envision several routes for improving the performance and the potential applicability of HiSMRT. For example, by employing an alternative dye that can effectively stain DNA in high ionic concentrations, we can more accurately quantify the concentration factor and recovery efficiency of HiSMRT near or even at physiological ionic concentrations. We also envision coupling HiSMRT to a wide variety of DNA analysis methods to demonstrate its versatility. Finally, a higher throughput could be achieved through multiplexing, increasing sample loading time, and optimizing the volumetric flow rate using larger channels. Given the demonstrated performance and potential for improvements, HiSMRT has the potential to concentrate low-abundant DNA biomarkers from physiologically relevant ionic concentrations for downstream detection and analysis. 


\section{Supplementary Material}

Refer to Web version on PubMed Central for supplementary material.

\section{ACKNOWLEDGMENTS}

The authors wish to thank Dr. Johan Melendez, Aniruddha Kaushik, and Pengfei Zhang for their great help in critical comments. We also gratefully acknowledge funding sources from the National Institutes of Health (Grants R44GM103356, R01AI137272, R01AI138978, R01AI117032, and U01CA214165).

\section{REFERENCES}

(1). Laird PW Nat. Rev. Genet 2010, 11 (3), 191-203. [PubMed: 20125086]

(2). Norton ME; Jacobsson B; Swamy GK; Laurent LC; Ranzini AC; Brar H; Tomlinson MW; Pereira L; Spitz JL; Hollemon D; Cuckle H; Musci TJ; Wapner RJ Obstet. Gynecol. Surv 2015, 70 (8), 483-484.

(3). Buckleton JS; Bright J-A; Taylor D Forensic DNA Evidence Interpretation; CRC Press, 2016.

(4). Aird D; Ross MG; Chen W-S; Danielsson M; Fennell T; Russ C; Jaffe DB; Nusbaum C; Gnirke A Genome Biol. 2011, 12 (2), R18. [PubMed: 21338519]

(5). Whitesides GM Nature 2006, 442 (7101), 368-373. [PubMed: 16871203]

(6). Yeo LY; Chang HC; Chan PPY; Friend JR Small 2011, 7 (1), 12-48. [PubMed: 21072867]

(7). Kelley SO ACS Sensors 2017, 2 (2), 193-197. [PubMed: 28723142]

(8). Wu Y; Tilley RD; Gooding JJ J. Am. Chem. Soc 2019, 141 (3), 1162-1170. [PubMed: 30463401]

(9). Kelley SO; Mirkin CA; Walt DR; Ismagilov RF; Toner M; Sargent EH Nat. Nanotechnol 2014, 9 (12), 969-980. [PubMed: 25466541]

(10). Kitagawa F; Otsuka KJ Chromatogr. A 2014, 1335, 43-60.

(11). Nge PN; Rogers CI; Woolley AT Chem. Rev 2013, 113 (4), 2550-2583. [PubMed: 23410114]

(12). Landers JP; Wen J; Legendre LA; Bienvenue JM Anal. Chem 2008, 80 (17), 6472-6479. [PubMed: 18754652]

(13). Datinská V; Voráčová I; Schlecht U; Berka J; Foret FJ Sep. Sci 2018, 41 (1), 236-247.

(14). Breadmore MC; Tubaon RM; Shallan AI; Phung SC; Abdul Keyon AS; Gstoettenmayr D; Prapatpong P; Alhusban AA; Ranjbar L; See HH; Dawod M; Quirino JP Electrophoresis 2015, 36 (1), 36-61. [PubMed: 25330057]

(15). Breadmore MC; Wuethrich A; Li F; Phung SC; Kalsoom U; Cabot JM; Tehranirokh M; Shallan AI; Abdul Keyon AS; See HH; Dawod M; Quirino JP Electrophoresis 2017, 38 (1), 33-59. [PubMed: 27678139]

(16). Breadmore MC; Grochocki W; Kalsoom U; Alves MN; Phung SC; Rokh MT; Cabot JM; Ghiasvand A; Li F; Shallan AI; Keyon ASA; Alhusban AA; See HH; Wuethrich A; Dawod M; Quirino JP Electrophoresis 2019, 40 (1), 17-39. [PubMed: 30362581]

(17). Chiu ML; Lawi W; Snyder ST; Wong PK; Liao JC; Gau V JALA 2010, 15 (3), 233-242.

(18). Lu Y; Liu T; Lamanda AC; Sin MLY; Gau V; Liao JC; Wong PK J. Lab. Autom 2015, 20 (6), 611-620. [PubMed: 25487557]

(19). Gabriel C; Peyman A; Grant EH Phys. Med. Biol 2009, 54 (16), 4863-4878. [PubMed: 19636081]

(20). Persat A; Marshall LA; Santiago JG Anal. Chem 2009, 81 (22), 9507-9511. [PubMed: 19831356]

(21). Khurana T; Bharadwaj R; Huber D; Santiago J In Handbook of Capillary and Microchip Electrophoresis and Associated Microtechniques, Third Edition 2007, 1085-1120.

(22). Garcia-Schwarz G; Bercovici M; Marshall LA; Santiago JG J. Fluid Mech 2011, 679, 455-475.

(23). Van Kooten XF; Truman-Rosentsvit M; Kaigala GV; Bercovici M Sci. Rep 2017, 7, 10467. [PubMed: 28874694] 
(24). Ye X; Mori S; Xu Z; Hayakawa S; Hirokawa T Electrophoresis 2013, 34 (22-23), 3155-3162. [PubMed: 24242290]

(25). Friedrich SM; Burke JM; Liu KJ; Ivory CF; Wang TH Nat. Commun 2017, 8 (1), 1213. [PubMed: 29089494]

(26). Liu KJ; Rane TD; Zhang Y; Wang TH J. Am. Chem. Soc 2011, 133 (18), 6898-6901. [PubMed: 21504160]

(27). Friedrich SM; Liu KJ; Wang TH J. Am. Chem. Soc 2016, 138 (1), 319-327. [PubMed: 26684193]

(28). Liu KJ; Wang T Biophys. J 2008, 95 (6), 2964-2975. [PubMed: 18515376]

(29). Martins D; Levicky R; Song YA Biosens. Bioelectron 2015, 72, 87-94. [PubMed: 25966462]

(30). Ault JT; Shin S; Stone HA J. Fluid Mech 2018, 854, 420-448.

(31). Shin S; Ault JT; Warren PB; Stone HA Phys. Rev. X 2017, 7 (4), 1-7.

(32). Shin S; Um E; Sabass B; Ault JT; Rahimi M; Warren PB; Stone HA Proc. Natl. Acad. Sci. U. S. A 2016, 113 (2), 257-261. [PubMed: 26715753]

(33). Lee D; Lee JA; Lee H; Kim SJ Sci. Rep 2019, 9 (1), 1-8. [PubMed: 30626917]

(34). Shin S; Ault JT; Feng J; Warren PB; Stone HA Adv. Mater 2017, 29 (30), 1-7.

(35). Lee JA; Lee D; Park S; Lee H; Kim SJ Sci. Rep 2018, 8 (1), 1-8. [PubMed: 29311619]

(36). Lee H; Kim J; Yang J; Seo SW; Kim SJ Lab Chip 2018, 18 (12), 1713-1724. [PubMed: 29796478]

(37). Florea D; Musa S; Huyghe JMR; Wyss HM Proc. Natl. Acad. Sci. U. S. A 2014, 111 (18), 6554 6559. [PubMed: 24748113]

(38). Musa S; Florea D; Wyss HM; Huyghe JM Soft Matter 2016, 12 (4), 1127-1132. [PubMed: 26616213]

(39). Vanýsek P Ionic Conductivity and Diffusion at Infinite Dilution In CRC Handbook of Chemistry and Physics, Ed. 95; Haynes WM, Ed.; CRC Press, 2014, pp 7779.

(40). Germann MW; Turner T; Allison SA J. Phys. Chem. A 2007, 111 (8), 1452-1455. [PubMed: 17269757]

(41). Friedrich SM; Bang R; Li A; Wang TH Anal. Chem 2019, 91, 2822-2830. [PubMed: 30668901]

(42). Liu KJ; Brock MV; Shih I; Wang TJ Am. Chem. Soc 2010, 132 (10), 5793-5798.

(43). Beh CW; Pan D; Lee J; Jiang X; Liu KJ; Mao HQ; Wang TH Nano Lett. 2014, 14 (8), 4729 4735. [PubMed: 25054542]

(44). Beh CW; Zhang Y; Zheng YL; Sun B; Wang TH Nucleic Acids Res. 2018, 46 (19), No. e117. [PubMed: 30010842]

(45). Foquet M; Korlach J; Zipfel W; Webb WW; Craighead HG Anal. Chem 2002, 74 (6), 1415-1422. [PubMed: 11922312]

(46). Wang X; Liu L; Pu Q; Zhu Z; Guo G; Zhong H; Liu SJ Am. Chem. Soc 2012, 134 (17), 7400_ 7405 .

(47). Stellwagen NC; Stellwagen EJ Chromatogr. A 2009, 1216 (10), 1917-1929. 


\section{Ionic Concentration (C) High Low}

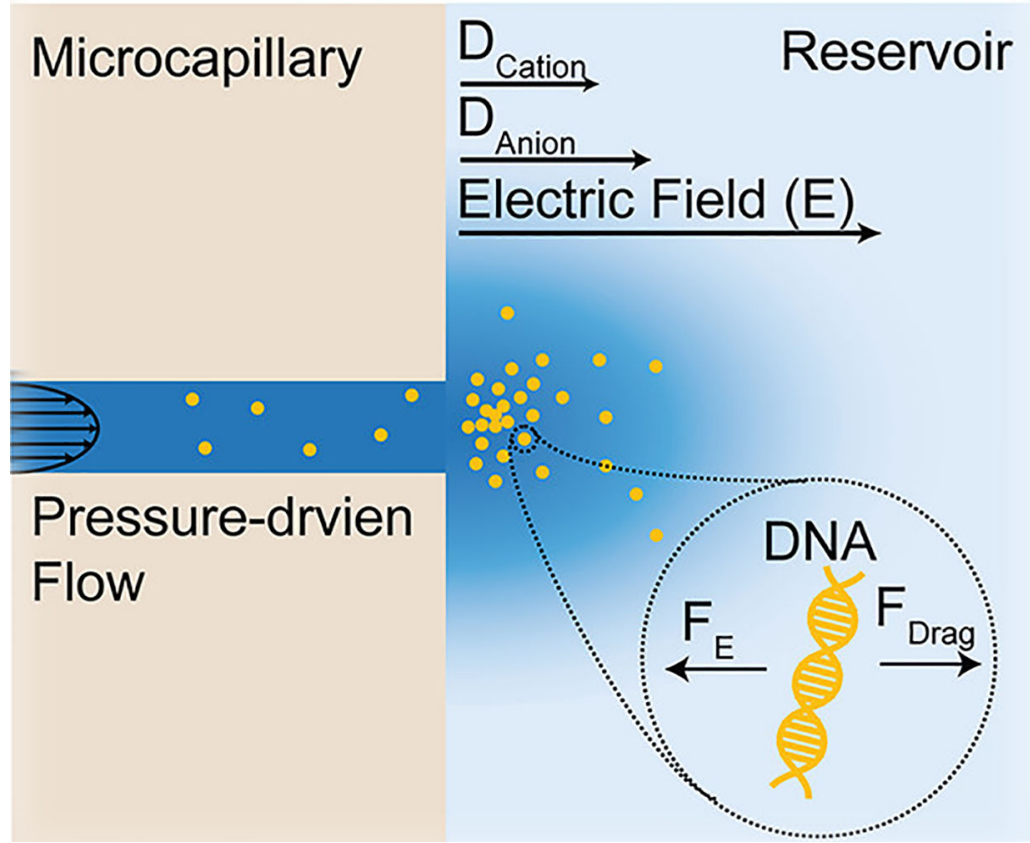

Figure 1.

Mechanistic underpinning of HiSMRT. Pressure-driven flow carries a low-concentration DNA sample in a high ionic concentration buffer through a microcapillary into a reservoir of low ionic concentration buffer, establishing a stable ion concentration gradient $(\nabla C)$ at the buffer interface. Since the ion diffusivity of the cation $\left(D_{\text {cation }}\right)$ is smaller than that of the anion $\left(D_{\text {anion }}\right)$ in the high-salt buffer, an electric field $(E)$ is induced as the ions diffuse into the reservoir. Thus, DNA can stably concentrate at the sample outlet where the DNA molecule experience balanced electrophoretic force $\left(F_{\mathrm{E}}\right)$ and the flow drag $\left(F_{\mathrm{Drag}}\right)$, without the presence of an external electric field. 
(a) HiSMRT

$\mathrm{t}=0 \mathrm{sec}$

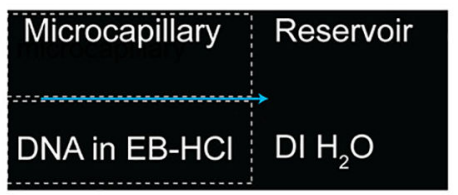

$\mathrm{t}=300 \mathrm{sec}$

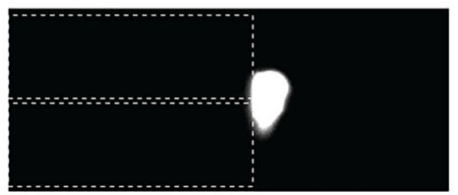

$\mathrm{t}=600 \mathrm{sec}$

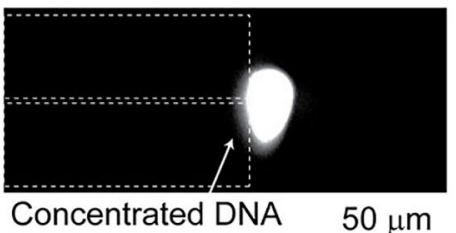

(b) No HiSMRT

$t=0 \mathrm{sec}$

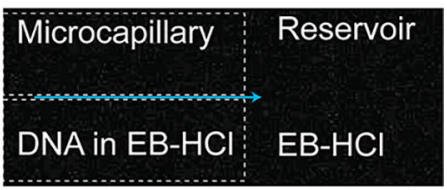

$\mathrm{t}=300 \mathrm{sec}$

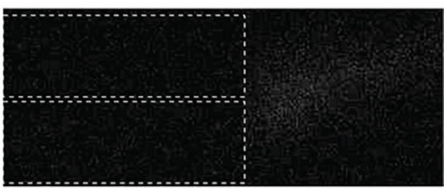

$\mathrm{t}=600 \mathrm{sec}$

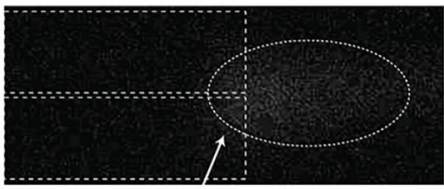

Dispersed DNA $50 \underline{\mu \mathrm{m}}$

Figure 2.

Microscopic snapshots of the DNA concentration process (a) with and (b) without HiSMRT. (a) Hind III digested $\lambda$ DNA sample in $1 \times \mathrm{EB}-\mathrm{HCl}$ buffer was pumped into a reservoir of deionized (DI) water. At the outlet of the microcapillary, DNA molecules concentrated into a bolus with a growing size within $600 \mathrm{~s}$ of observation. (b) DI water was replaced by the $1 \times$ $\mathrm{EB}-\mathrm{HCl}$ buffer to eliminate the ionic concentration. The absence of the ion concentration gradient resulted in dispersed DNA in the downstream of the DNA sample outlet without concentration (as shown in the dotted circle). This comparison confirms that an ionic concentration gradient is necessary for the HiSMRT DNA concentration. 
(a)

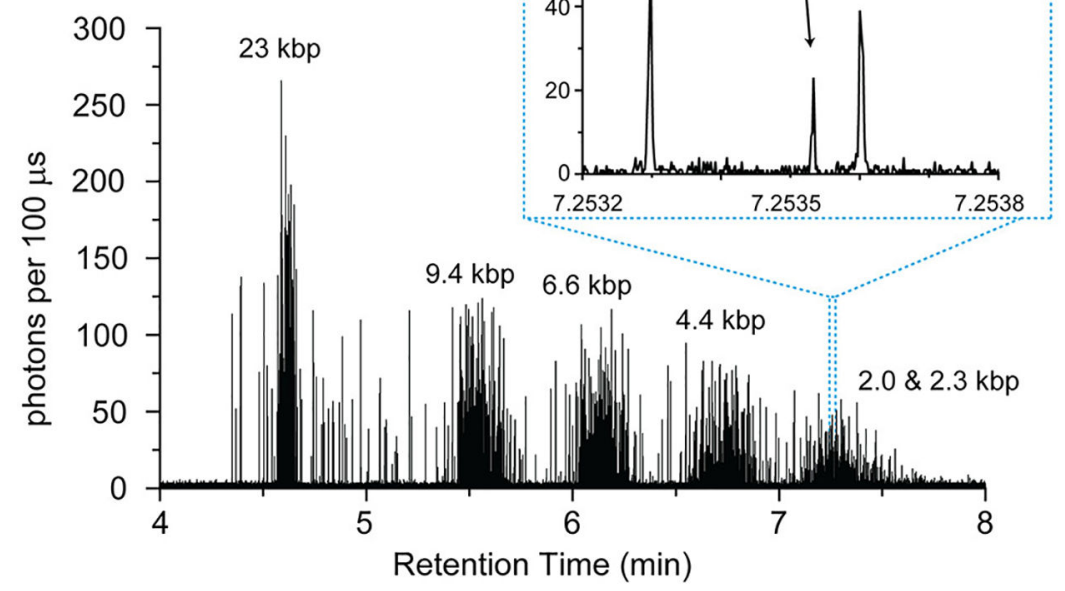

(b)

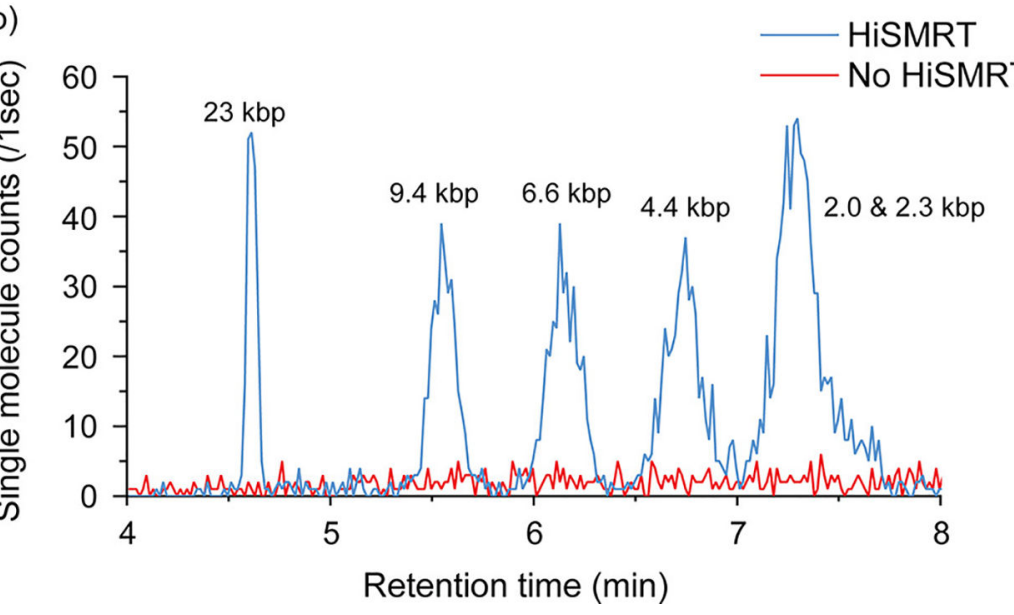

Figure 3.

HiSMRT concentration experiment coupled with single-molecule-free solution hydrodynamic separation (SML-FSHS) on the cylindrical illumination confocal spectroscopy (CICS) platform for quantitative analysis of Hind III digested $\lambda$ DNA. (a) Time trace of the single-molecule fluorescence bursts of DNA concentrated by HiSMRT, separated by SML-FSHS, and detected by CICS. (b) Free solution hydrodynamic separation chromatograms obtained by the single-molecule counting analysis provides quantitative capabilities for the concentration performance. It shows that many DNA molecules are detected with HiSMRT, while only a low level of random noise was detected without HiSMRT. These results confirm that HiSMRT indeed concentrated DNA and verify the observation on the microscope in Figure 2. 
(a)

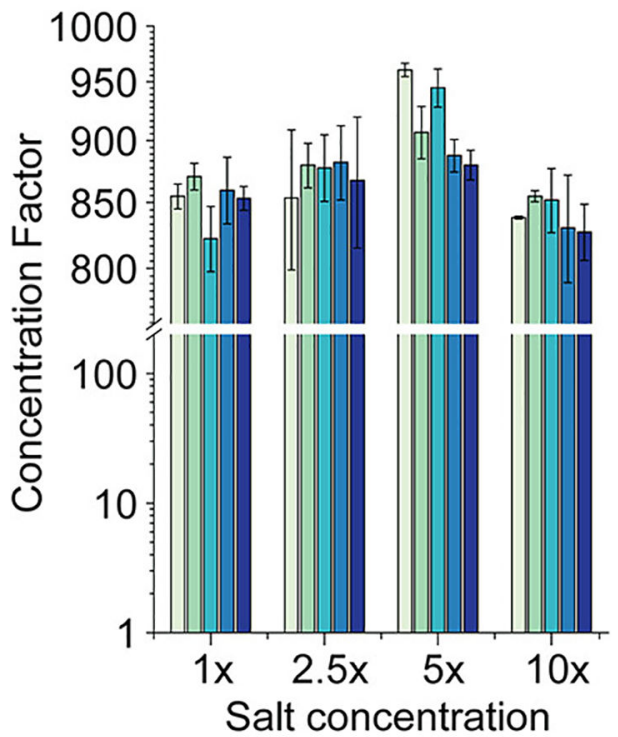

(b)

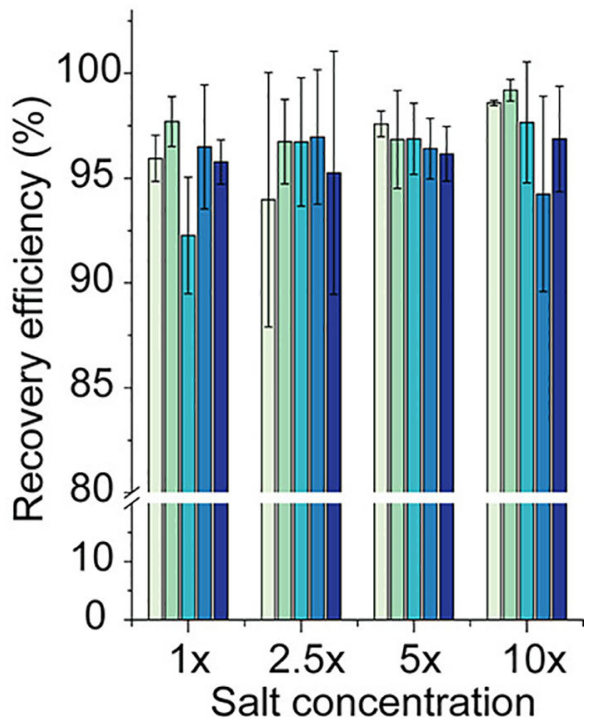

\section{$\square 2.0$ \& 2.3 kbp $\square 4.4$ kbp $\square 6.6$ kbp $\square 9.4$ kbp $\square 23$ kbp}

Figure 4.

Performance of HiSMRT remains high across 10-fold high ionic concentrations ranging up to $10 \times \mathrm{EB}-\mathrm{HCl}$ (equivalent to $20 \%$ blood ionic concentration) with minimal DNA size bias. In the best case, a 960-fold concentration factor and $97.6 \%$ recovery efficiency were achieved (for the 2.0 and $2.3 \mathrm{kbp}$ fragment). These results clearly demonstrate that HiSMRT is robust in at least $10 \times \mathrm{EB}-\mathrm{HCl}$ buffer and consistent across a 10 -fold ionic concentration range. Error bars show the standard deviations of triplicate experiments at each condition. 

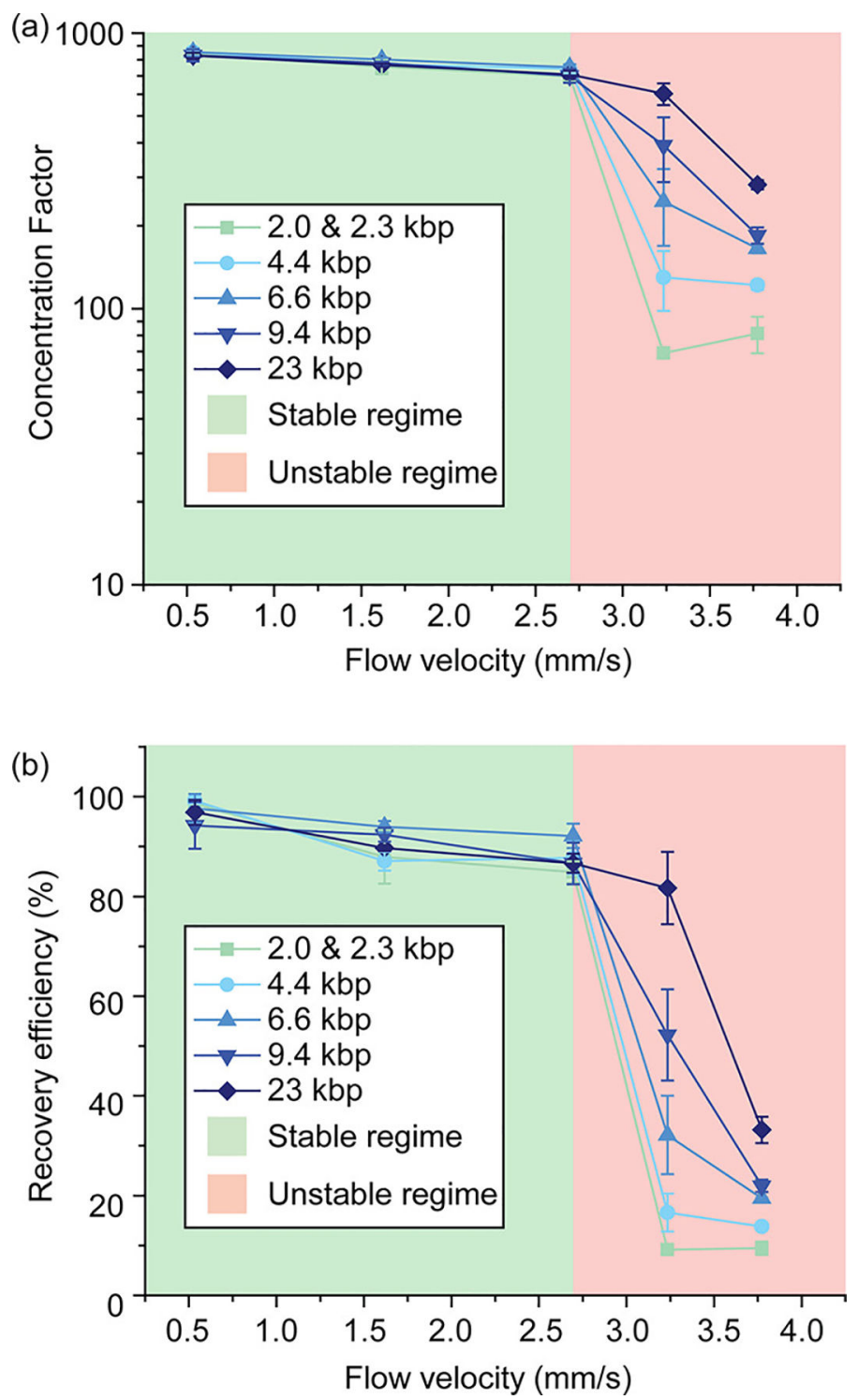

Figure 5.

Flow velocity dictates the performance of HiSMRT: In stable regime (green), the performance remains steadily high, insensitive to velocity increase with minimal variation across the DNA fragments from 2.0 to $23 \mathrm{kbp}$. On the contrary, in the unstable regime (red), the performance drops sharply with increasing velocity and shows large variation across different DNA sizes. Error bars show the standard deviations of triplicate experiments at each condition. $10 \times \mathrm{EB}-\mathrm{HCl}$ was used as the high ionic concentration buffer. 